\title{
MIOCARDITE AGUDA SOB A FORMA DE SÍNDROME CORONARIANA AGUDA APÓS INFECÇÃO DO TRATO URINÁRIO
}

\author{
ACUTE MYOCARDITIS PRESENTING AS ACUTE CORONARY \\ SYNDROME FOLLOWING URINARY TRACT INFECTION
}

\author{
Guilherme Dagostin de Carvalho', Stefano Boemler Busato', \\ Fernando Pivatto Júnior', Murilo Foppa², Solano Vinícius Berger², \\ Miguel Gus ${ }^{2}$
}

\begin{abstract}
RESUMO
A miocardite é cada vez mais diagnosticada, principalmente pela maior disponibilidade de métodos como a ressonância magnética cardíaca. A apresentação clínica é variável, geralmente posterior a uma infecção respiratória ou gastrointestinal, manifestando-se como síndrome coronariana aguda (SCA), insuficiência cardíaca aguda ou crônica, arritmias cardíacas ou mesmo choque cardiogênico inexplicável. Relatos de casos de miocardite após infecção do trato urinário (ITU) são escassos. Neste relato, descrevemos o caso de um paciente masculino de 24 anos com miocardite após ITU que se apresentou sob a forma de SCA.
\end{abstract}

Palavras-chave: Miocardite; infecções urinárias; síndrome coronariana aguda

\section{ABSTRACT}

The diagnosis of myocarditis has increased mainly due to greater availability of methods such as cardiac magnetic resonance (CMR). Its clinical presentation varies, usually following respiratory or gastrointestinal tract infection, in patients presenting with acute coronary syndrome (ACS), acute or chronic heart failure, cardiac arrhythmias, or even unexplained cardiogenic shock. Case reports of patients with myocarditis following urinary tract infection (UTI) are scarce. This is a case report of a 24-year-old male patient with myocarditis with symptoms of ACS following UTI.

Keywords: Myocarditis; urinary tract infections; acute coronary syndrome

A miocardite é cada vez mais diagnosticada, principalmente pela maior disponibilidade de métodos investigativos como a ressonância magnética cardíaca (RMC). Sua etiologia vai desde agentes infecciosos, principalmente os virais, até agentes tóxicos e imunológicos. A apresentação clínica é variável, geralmente posterior a uma infecção respiratória ou gastrointestinal, manifestando-se como síndrome coronariana aguda (SCA), insuficiência cardíaca (IC) aguda ou crônica, arritmias cardíacas ou mesmo choque cardiogênico inexplicável ${ }^{1-3}$.

A miocardite bacteriana apresenta um campo em aberto por se tratar de uma doença rara, com poucos estudos sobre sua fisiopatologia e casos confirmados com realização de biópsia miocárdica ou necropsia. Os mecanismos de lesão propostos incluem invasão bacteriana direta, mediada por toxinas ou resposta imune ${ }^{4-6}$. Neste relato, descrevemos o caso de um paciente masculino de 24 anos com miocardite que se apresentou sob a forma de SCA após infecção do trato urinário (ITU).
Clin Biomed Res. 2017;37(1):51-54

1 Serviço de Medicina Interna, Hospital de Clínicas de Porto Alegre (HCPA), Porto Alegre, RS, Brasil.

2 Serviço de Cardiologia, Hospital de Clínicas de Porto Alegre (HCPA), Porto Alegre, RS, Brasil.

Autor Correspondente: Guilherme Dagostin de Carvalho dagostinguilherme@gmail.com Hospital de Clínicas de Porto Alegre (HCPA), Rua Ramiro Barcelos, 2350. 90035-903, Porto Alegre, RS, Brasil. 


\section{RELATO DO CASO}

Homem de 24 anos, branco, ex-tabagista (10 anos/maço), procurou o serviço de emergência com relato de disúria há cinco dias, associada a dor lombar, náuseas, vômitos e febre não aferida. $\mathrm{Na}$ admissão, apresentava sinais vitais estáveis e exame clínico normal. Foi coletado exame qualitativo de urina com identificação de piúria, nitrito e esterase leucocitária, sendo então iniciado tratamento oral com ciprofloxacino $500 \mathrm{mg}$ de 12/12 horas.

Após três dias, teve desconforto retroesternal progressivo e procurou atendimento em serviço de emergência. Como história familiar para doença cardiovascular, apresentava mãe hipertensa e irmão com morte súbita aos 30 anos de idade. Negou uso de drogas ilícitas ou medicamentos exceto o antimicrobiano prescrito. Foi realizado eletrocardiograma (ECG), que identificou supradesnivelamento do segmento ST em parede inferior (figura 1). Iniciou-se manejo para SCA com dupla antiagregação plaquetária em dose de ataque (ácido acetilsalicílico $300 \mathrm{mg}$ e clopidogrel $600 \mathrm{mg}$ ) e anticoagulação com enoxaparina, e foi solicitada transferência para hospital com serviço de hemodinâmica disponível. Nos exames laboratoriais, observava-se troponina I de $17,63 \mathrm{ng} / \mathrm{mL}$ (referência: <0,16), 3.980 leucócitos (2.350 segmentados e 1.080 linfócitos), sorologias virais para vírus da imunodeficiência humana (HIV) e hepatites $B$ e $C$ negativas. Raio $X$ de tórax estava normal. Foi conduzido 4 horas após a piora da dor para cineangiocoronariografia, que revelou coronárias isentas de lesões ateromatosas significativas.

No dia seguinte, apresentou febre, sem outros sinais ou sintomas associados. Pela suspeita clínica de miocardite, foi submetido dois dias depois a RMC (figura 2), que confirmou a hipótese diagnóstica. O paciente apresentou boa evolução, sem desenvolvimento de IC, permanecendo apenas com desconforto retroesternal após o início de anti-inflamatórios. Troponina I teve pico em $19,5 \mathrm{ng} / \mathrm{mL}$, com queda para $2,83 \mathrm{ng} / \mathrm{mL}$ após dois dias. Proteína C-reativa decaiu de 90 para 20 mg/L concomitantemente ao descenso da troponina e uso de anti-inflamatórios. Uroculturas e hemoculturas foram negativas, mas o paciente estava em uso de antibiótico há cinco dias. Teve alta com diagnóstico de miopericardite após ITU, recebendo anti-inflamatório e antibiótico por 10 dias.

\section{DISCUSSÃO}

A miocardite é uma condição inflamatória do músculo cardíaco que pode acometê-lo de maneira focal ou difusa e, em alguns casos, conjuntamente afetar o pericárdio. Ela apresenta diversos gatilhos em sua etiologia, desde infecciosos - com destaque aos vírus, mas também bactérias, fungos, helmintos, protozoários, riquétsias e espiroquetas - até autoimunes (lúpus eritematoso sistêmico, granulomatose com
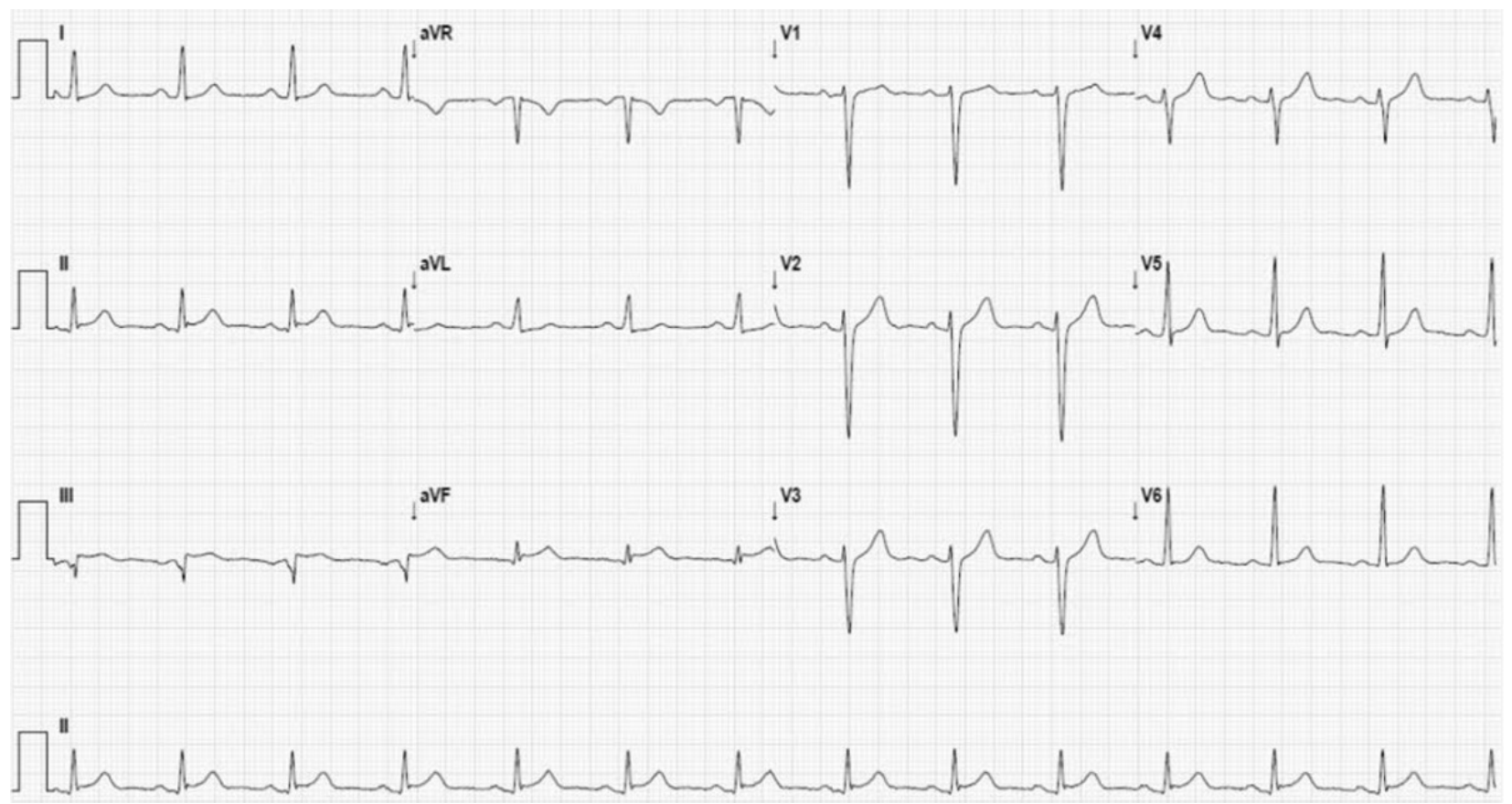

Figura 1: Eletrocardiograma com supradesnivelamento do segmento ST em parede inferior (D3 e aVF). 


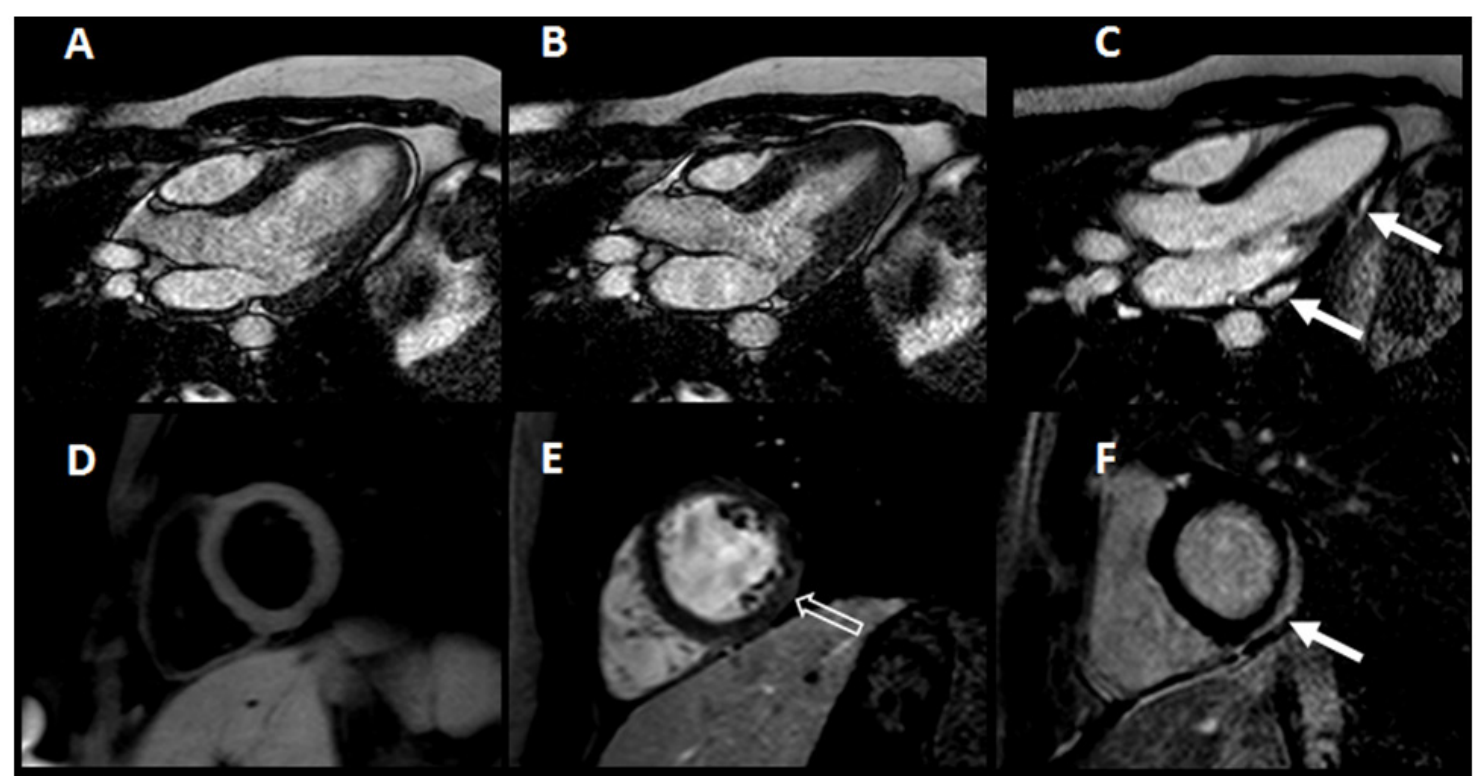

Figura 2: Ressonância magnética cardíaca mostra função sistólica normal nas imagens b-SSFP dinâmicas em 3 câmaras na diástole $(A)$ e na sístole $(B)$ e realce tardio de gadolínio $(C)$ subepicárdico e pericárdico nos segmentos infero-latero-basal e infero-latero-medial (setas brancas). Imagens no eixo transversal mostram edema pelo aumento difuso da intensidade do sinal nas imagens ponderadas em T2 (D), realce precoce (E) no segmento infero-latero-medial (seta oca) e realce tardio subepicárdico e pericárdico de gadolínio (F) no segmento infero-latero-basal (seta). Achados sugestivos de miopericardite.

poliangeíte, etc.), além de hipersensibilidade a medicamentos e/ou drogas ilícitas, radioterapia, intoxicação por metais pesados, endocrinopatias, entre outros. Tem também associação com o HIV. Em estudo realizado por pesquisadores da África do Sul, $44 \%$ dos pacientes com cardiomiopatia associada ao HIV apresentavam miocardite, sendo que infecção por vírus cardiotrópicos estava presente em todos esses ${ }^{1-3,7}$.

Relatos de casos de miocardite após ITU são escassos, com apenas cinco casos até o momento, todos secundários a Escherichia coli. Uma explicação plausível para essa crescente associação é o poder invasivo e de produção de toxinas dos diversos gêneros da bactéria ${ }^{4-6,8,9}$.

Não há consenso quanto ao diagnóstico de miocardite. A biópsia endomiocárdica é o padrão-ouro, porém apresenta riscos inerentes e não está disponível na maioria dos serviços, o que inviabiliza o seu uso rotineiro. Para facilitar o reconhecimento na prática clínica e a seleção de pacientes que mereçam investigação pormenorizada, a European Society of Cardiology propõe um critério diagnóstico. Ele se baseia em cinco apresentações clínicas (dor torácica pleurítica/anginosa; surgimento/piora aguda de sinais/sintomas de IC; piora subaguda/crônica dos mesmos; palpitações, síncope ou morte súbita abortada; choque cardiogênico) e em quatro alterações de exames complementares (ECG ou Holter; elevação de marcadores de necrose miocárdica; alteração funcional e/ou estrutural ao ecocardiograma ou RMC; achados sugestivos na RMC, de acordo com os critérios de Lake-Louise). O preenchimento de um critério clínico e um complementar (ou dois complementares nos pacientes que estejam assintomáticos no momento da avaliação), na ausência de estenose coronariana $>50 \%$ e cardiopatia prévia ou atual que justifique o quadro, configura um caso suspeito de miocardite ${ }^{10}$.

A elevação de biomarcadores cardíacos refletindo necrose miocárdica é vista apenas em alguns pacientes com miocardite. Achados clínicos e experimentais sugerem que a elevação das troponinas I ou T é mais comum que da isoenzima CK-MB. O aumento dos níveis séricos de troponina I correlaciona-se com uma curta duração de sintomas de IC, sugerindo que a necrose miocárdica ocorre precocemente no curso da doença. Elevações persistentes de biomarcadores sugerem necrose em curso ${ }^{11,12}$.

O tratamento deve ser direcionado para a etiologia. Entretanto, o efeito de terapias específicas foi confirmado por poucos estudos e apenas em doenças inflamatórias, como a sarcoidose e a miocardite de células gigantes. Devido à grande incidência de disfunção ventricular, o manejo da IC é mandatório nesses pacientes. Anti-inflamatórios não esteroidais (AINEs) e colchicina são utilizados na terapêutica da pericardite, mas não há indicação 
para o uso dos mesmos em pacientes com miocardite. Em estudos com murinos, AINEs aumentaram o processo inflamatório e a mortalidade no cenário de miocardite viral aguda. Assim, o uso deles na dose mínima necessária para o alívio da dor fica reservado aos pacientes com miopericardite com função ventricular preservada ${ }^{1-3}$.

Escherichia coli é o bacilo gram-negativo responsável pela maior diversidade de infecções extraintestinais e é causa comum de bacteremia, possuindo cepas comensais e patogênicas. Em estudos prévios, as bactérias isoladas no trato urinário possuíam mais fatores de virulência, como fímbrias que facilitam a aderência e invasão tecidual, bem como mais lipopolissacarídeos, que induzem resposta do hospedeiro mediada por citocinas inflamatórias e fator de necrose tumoral, levando a dano miocárdico via receptor toll-like. Também produz diversas toxinas, entre elas a $\beta$-hemolisina - que está associada à rápida perda da viabilidade e função de cardiomiócitos em infecções estreptocócicas - e serina proteases específicas que danificam canais de potássio das células miocárdicas ${ }^{4-6}$.

No caso apresentado, a infecção urinária provavelmente foi causada pela bactéria Escherichia coli, a mais comumente associada ${ }^{13}$. O diagnóstico de miocardite após ITU é presuntivo, tendo em vista que a confirmação é dada pela biópsia com identificação da bactéria. Optou-se por não realizar esse procedimento devido aos riscos inerentes, à boa evolução clínica e ao fato de não alterar o curso clínico/tratamento da doença.

\section{REFERÊNCIAS}

1. Kindermann I, Barth C, Mahfoud F, Ukena C, Lenski M, Yilmaz A, et al. Update on myocarditis. J Am Coll Cardiol. 2012;59:779-92.

2. Cooper LT JR. Myocarditis. N Engl J Med. 2009;360:1526-38.

3. Sagar S, Liu PP, Cooper LT Jr. Myocarditis. 2012;379:738-47.

4. Uribarri A, Martínez-Sellés M, Yotti R, Pérez-David $E$, Fernández-Avilés $F$. Acute myocarditis after urinary tract infection by Escherichia coli. Int $J$ Cardiol. 2011;152:e33-4.

5. Chen TC, Lu PL, Lin CY, Lin WR, Chen $\mathrm{YH}$. Escherichia coli urosepsis complicated with myocarditis mimicking acute myocardial infarction Am J Med Sci. 2010;340:332-4.

6. Jang L, Wu C, Chen $\mathrm{H}$, Chen $Y$. Myocarditis in a patient with Escherichia coli urinary tract infection. Acta Neurol (Napoli). 2011;25:192-5.
7. Shaboodien G, Maske C, Wainwright $\mathrm{H}$, Smuts H, Ntsekhe M, Commerford PJ, et al. Prevalence of myocarditis and cardiotropic virus infection in Africans with HIV-associated cardiomyopathy, idiopathic dilated cardiomyopathy and heart transplant recipients: a pilot study: cardiovascular topic. Cardiovasc J Afr. 2013;24:21823.

8. Gentile G, Meles E, Carbone C Gantú E, Maggiolini S. Unusual case of myocardial injury induced by Escherichia coli sepsis. Monaldi Arch Chest Dis. 2010;74:40-3.

9. Abu-Arafeh I, Gray E, Youngson G, Auchterlonie I, Russell G. Myocarditis and haemolytic uraemic syndrome. Arch Dis Child. 1995;72:46-7.

10. Caforio AL, Pankuweit S, Arbustini E, Basso C, Gimeno-Blanes J, Felix $\mathrm{SB}$, et al. Current state of knowledge on aetiology, diagnosis, management, and therapy for myocarditis: a position statement of the European Society of Cardiology Working Group on Myocardial and Pericardial Diseases. Eur Heart J. 2013;34(33):2636-48, 2648a-2648d.

11. Smith SC, Ladenson JH, Mason JW, Jaffe AS. Elevations of cardiac troponin I associated with myocarditis. Experimental and clinical correlates. Circulation. 1997;95:163-8.

12. Lauer B, Niederau C, Kühl U, Schannwell M, Pauschinger M, Strauer BE, et al. Cardiac troponin T in patients with clinically suspected myocarditis. J Am Coll Cardiol. 1997;30:1354-9.

13. Czaja CA, Scholes D, Hooton TM, Stamm WE. Population-based epidemiologic analysis of acute pyelonephritis. Clin Infect Dis. 2007;45:273-80. 